\title{
Effect of starvation on expression of troponin complex genes and ER stress associated genes in skeletal muscles.
}

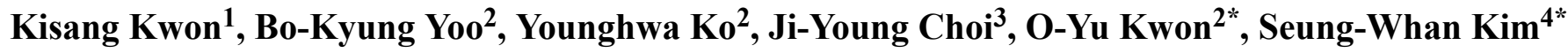 \\ ${ }^{1}$ Department of Biomedical Laboratory Science, College of Health and Welfare, Kyungwoon University, Gumi 39160, \\ Korea \\ ${ }^{2}$ Departments of Medical Science and Anatomy and Cell Biology, College of Medicine, Chungnam National University, \\ Daejeon 34134, Korea \\ ${ }^{3}$ Applied Entomology Division, National Academy of Agricultural Science, RDA, Wanju 55365, Korea \\ ${ }^{4}$ Department of Emergency Medicine, College of Medicine, Chungnam National University, Daejeon35015, Korea
}

\begin{abstract}
Little is known about the mechanism of gene expression changes in skeletal muscle during starvation. This study investigated the influence of starvation on gene expression of troponin complex (TnC, TnI and TnT), tropomyosin (Tpm), and endoplasmic reticulum (ER) stress associated proteins (BiP, calreticulin, PDI, and ATF6) in three kinds of skeletal muscles of Gryllus bimaculatus. Dorsal ventral flight muscle (DVM), dorsal longitudinal, and dorsal wing flight muscles (DWM) were identified as typical skeletal muscles by H\&E staining. Obvious gene expression was detected in the DVM, but not in the DLM or DWM. In addition to gene expression in DVM, gene expression of decorin, a type of myokine, was also significantly increased by starvation. This is the first study that reports the effect of starvation on expression of troponin complex genes and ER stress associated genes in three types of skeletal muscles.
\end{abstract}

Keywords: Gryllus bimaculatus, Starvation, Troponin complex (TnC, TnI and TnT), Tropomyosin (Tpm), Endoplasmic reticulum (ER) stress associated proteins (BiP, calreticulin, PDI and ATF6).

Accepted on April 26, 2018

\section{Introduction}

Two-spotted cricket Gryllus bimaculatus (Orthoptera: Gryllidae), an hemimetabolous insect species, is one of the most economically significant cricket species in the world. It is used as feed for poultry and fish [1]. It can be easily bred outdoors. Recently, it has been used as a model insect for tissue regeneration, neurophysiology, and behavioral research in biomedical field [2].

Muscles in the body are divided into three major types: skeletal, cardiac, and smooth. Among them, skeletal muscles are the most abundant tissues. They mainly function by muscle contraction-relaxation activation. As part of the endocrine system, skeletal muscles produce various myokines associated with metabolism, growth, and disease [3,4]. Histologically, skeletal muscles contain a network of sarcoplasmic reticulum (the endoplasmic reticulum, ER) that plays a significant role in maintaining homeostasis [5]. Globular actin monomers can polymerize to filamentous actin to form a double-helical structure that is longitudinally oriented with tropomyosin $(\mathrm{Tm})$ [6]. One troponin complex binds to every seventh actin monomer. The complex has three regulatory proteins: troponin
$\mathrm{C}$, troponin $\mathrm{C}$, and troponin $\mathrm{T}$. Troponin $\mathrm{C}(\mathrm{TnC})$ binds to a calcium molecule [7]. Troponin I (TnI) is the inhibitory subunit while troponin $\mathrm{T}(\mathrm{TnT})$ binds to $\mathrm{Tm}[8,9]$.

During skeletal muscle contraction, calcium binds to $\mathrm{TnC}$ and induces conformational change of troponin complex to $\mathrm{Tm}$ that allows binding for actin-myosin complex and muscle contraction-relaxation [10]. Insects only have skeletal muscles. They do not have smooth or cardiac muscles. The two-spotted cricket G. bimaculatus has three different kinds of skeletal muscles observed by ventral dissection: dorsal longitudinal flight muscle (DLM), dorsal ventral flight muscles (DVM), and dorsal wing flight muscles (DWM) [11].

Endoplasmic reticulum (ER) comprises a sophisticated signal transducing system for maintaining cellular homeostasis through post-translational modification of secretory proteins [12]. Stress in the ER is induced by unfolded protein response (UPR) to adaption and survival of cells and/or tissues by expressing ER chaperones such as binding immunoglobulin protein (BiP), calreticulin, and protein disulfide isomerase (PDI). ER chaperones are regulated directly or indirectly by ER transmembrane sensors such as inositol-requiring enzyme 1 
(IRE1), protein kinase-like ER Kinase (PERK), and activating transcription factor 6 (ATF6) [13,14].

Starvation is one of the most common stresses that insects encounter. It especially has serious effects on small-bodied insects. Thus, it is essential for insects to adapt to starvation by metabolism through various mechanisms, including gene regulation and enzyme (protein) modification. It is known that starvation influences expression levels of several genes such as allatotropin in the oriental armyworm, Mythimna separata, different mitochondrial genes in the mosquito, Aedes aegypti, and tissue-specific genes in the wood ant, Formica exsecta [15-17]. Starved insects are more susceptible to pathogens due to their compromised immune systems (e.g., Colorado potato beetle, Leptinotarsa decemlineata) [18]. During starvation, differential gene expression in skeletal muscles has been reported in vertebrates, with ER-stress associated gene as a potential mediator of anabolic resistance [19].

The objective of this study was to investigate changes in gene expression that occur in skeletal muscle from starvation. We investigated the effect of starvation on gene expression in troponin complex (TnC, TnI, TnT, and Tm) and ER-stress associated genes (BiP, calreticulin, PDI and ATF6) in skeletal muscles (DLM, DVM, and DWM) of G. bimaculatus. Results of this study will provide important information for understanding effects of starvation on physiological changes through gene expression regulation of various skeletal muscles.

\section{Materials and Methods}

\section{Insects and H\&E staining}

Normal cricket rearing basically followed Dr. Nagata's method [20]. Fifth-instar larvae of the two-spotted cricket $G$. bimaculatus used in this study were obtained from the Rural Development Administration of Korea (Jeonju, South Korea). Only male adults were reared at $28-30^{\circ} \mathrm{C}$ with humidity of $70 \%$ under a $10 \mathrm{~h} / 14 \mathrm{~h}$ light/dark photoperiod in transparent plastic cylinders (diameter of $9 \mathrm{~cm}$ and height of $10 \mathrm{~cm}$ ). Crickets were fed a commercial feed suited for rats and rabbits (1:6) with unlimited water supply. Crickets with synchronous growth from the fifth instar to adult emergence were selected. Only males were used in the following experiments. Due to cannibalism, each vial contained only a male cricket. For starvation classification, crickets were allowed free access to water. However, they were not fed for three to five days. Five crickets were anesthetized by exposure to $\mathrm{CO}_{2}$ gas for dissection. To observe muscle tissues, specimens were processed for hematoxylin and eosin (H\&E) staining according to standard protocols [21] and analyzed by virtual microscopy (Nikon Eclipse E600). Insects of the control group were reared under normal breeding conditions as described above until the following experiments.

\section{RNA isolation and RT-PCR}

Each tissue section was removed from each of five insects under dissecting microscope (Olympus SZ51), placed into a
$1.5 \mathrm{ml}$ tube containing $100 \mu \mathrm{l}$ TRIzol reagent (Invitrogen, Carlsbad, CA, USA), and homogenized using a plastic pestle matched to $1.5 \mathrm{ml}$ Tube (SP Scienceware, Wayne, NJ, USA). Total RNA was extracted using TRIzol reagent according to the manufacturer's instruction, quantified with a NanoDrop Lite UV-spectrophotometer (Thermo Scientific, Waltham, MA, USA) and reverse-transcribed to cDNA using a SuperscriptII ${ }^{\mathrm{TM}}$ First Strand Synthesis Kit (Invitrogen Carlsbad, CA, USA). The resulting cDNA was PCR-amplified using primer pairs for cricket actin (cActin) F ATCACTGCCCTTGCTCCTTC-3') and $\mathrm{R}$ (5'TTCCTGTGGACAATGGATGG-3'), cTnI F (5'TGAGAGGAGACGCATCATTG-3') and cTnI R (5'CTGCCTTCTCGGATTTCTTG-3') and, cTnC F (5'GAGAAGAGCGGTTCCATCAG-3') and cTnC R (5'AAGCTTCGCGTAACTCTTGC-3'), cTnT F (5'GCAAGCAAAGGTCAAAGGAG-3') and cTnT R (5'GGCTTGACGTTTCCTCTCAG-3'), cTpm F (5'ACCCTGAGCGTCAAGCTAAA-3') and cTpm R (5'GGAGTCCAAATCGTCCGTAA-3'), cDecorin F (5'GGCTTGGAATCACATCACAATC-3') and cDecorin R (5'AGCCTCAGCAACGTCAAA-3'), cATF6 F (5'ACTTTGCGCCCTAAGATG-3') and cATF6 F (5'GTGATGGAGCTGATGGTTT-3'), cBiP F (5'GCTTTGTGCCTCCTTTATGT-3') and cBiP R (5'AAGGCGTAATTCTATTGCCC-3'), cCalr F (5'CGTCTTGTCTGAGTTCGTG-3') and cCalr R (5'GGGTGCTCAGAATAAATCCA-3'), and cPDI F (5'CAGTACAATCCACTTTGGCA-3') and cPDI R (5'CGGAACATGAAACTGTGCTA-3'). RT-PCR was performed with 30 cycles of $94^{\circ} \mathrm{C}$ for $30 \mathrm{sec}, 58^{\circ} \mathrm{C}$ for $30 \mathrm{sec}$, and $72^{\circ} \mathrm{C}$ for one minute (10 $\mathrm{min}$ in the final cycle), using gene-specific primer set (forward and reverse primers) listed above with Taq DNA polymerase. Results of a representative experiment from three experiments with different samples are shown.

\section{Results and Discussion}

When ventral aspect was observed under a light microscope, two milk-tinted DLMs in an elongated triangular shape with the apex attached to a keel-like phragma protruding from the anterior margin of the pterothorax were found (Figure 1A). Major tracheal trunks were located at the ventral margin of DLMs. Whitish columnar shaped DVMs ran vertically against DLMs to generate power for wings' upstroke by contraction. DWMs were pinkish compared to the other two types of muscles (DLMs and DVMs). They ran parallel directly above DLMs. Histological features of muscle groups were observed after H\&E staining. Skeletal muscle fibers were cut longitudinally and transversely (Figures 1B-1D). Three types of muscles exhibited commonly typical skeletal features histologically, with peripheral nuclei lying just under the sarcolemma. Among them, DVM had a specific empty space between dense muscle bundles to reduce total weight of the muscle for flight.

Animals respond to starvation by searching for new food resources or by reducing energy consumption. Although 
animals do not die initially at the onset of starvation, they undergo physiological changes until mortality. To overcome starvation, insects have developed a mechanism to convert fat decomposition into energy source through muscle proteolysis [22]. Proteolysis is primarily responsible for increased skeletal muscle protein breakdown during starvation and the resulting amino acids can be used to synthesize new proteins required for survival to maintain normal several pathways [23-25]. Insects that are relatively small in body size are sensitive to environmental changes. They have developed unique survival mechanisms.
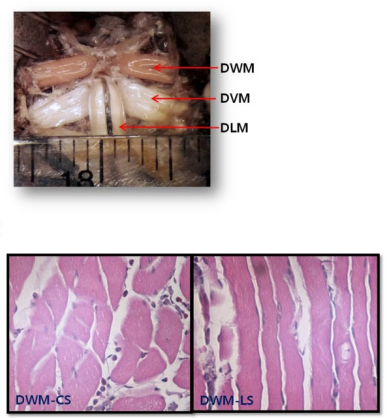
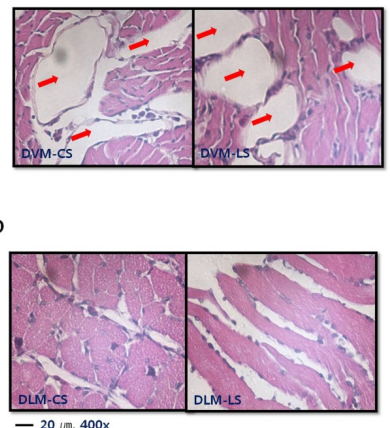

Figure 1. Anatomical and histological features of cricket skeletal muscle. (A) Ventral dissection of a cricket to reveal its gross anatomy, showing three kinds of skeletal muscles: dorsal longitudinal flight muscles (DLMs), dorsal ventral flight muscles (DVMs), and dorsal wing flight muscles (DWMs). Scale: $1 \mathrm{~mm}$. (B) Cross section of DVM (DVM-CS) (left panel) and longitudinal section of DVM-LS (right panel). Arrows indicate specific empty spaces. (C) Cross section of $D W M(D W M-C S)$ (left panel) and longitudinal section of DWM-LS (right panel). (D) Cross section of DLM (DLM-CS) (left panel) and longitudinal section of (DLM-LS) (right panel). Figures $B, C$, and $D$ were observed at $400 X$ magnification.

To understand changes of gene expression in skeletal muscles induced by starvation, expression levels of troponin complexes genes (TnI, TnC and TnT) and Tmp in G. bimaculatus skeletal muscles (DLM, DVM, and DWM) were determined. During starvation, there were no significant changes in gene expression in DLM or DWM (Figures 2B-2C). However, there were significant changes in gene expression in the DVM (Figure 2A). Levels of gene expression were gradually increased more than two-fold after five days of starvation compared to those in the control group. It was unclear why high levels of gene expression induced by starvation only occurred in the DVM. Based on anatomical position, the DLM is not exposed or sensitive to environmental changes because it functions like a skeleton that maintains body form. The DWM is directly attached to the wings and its function is flight related. DVM is a specialized muscle that responds conservatively to metabolic changes to maintain energy reserves and avoid predators by flight.

Glucose in the feed is the most significant substrate for ATP biosynthesis and glycosylation of proteins through posttranslational modification in the ER. To maintain ER homeostasis, UPR as one of specific pathways is commonly developed in eukaryotic cells. It involves three distinct ER transmembrane sensors (IRE1, PERK, and ATF6) that are downstream components of ER lumen chaperones [26,27]. Activation of IRE1 (autophosphorylation and dimerization) that regulates cleavage of X-box DNA-binding protein (XBP) mRNA will produce an activated form of XBP1 and activate PERK. Activation of PERK then induces phosphorylation of the $\alpha$ sub-unit of eukaryotic translation initiation factor 2 (eIF2 $\alpha$ ) and ATF6 that is cleaved by ER stress. This action results in N-terminal cytoplasmic domain binding to ER stressresponse element (ERSE) and ATF6 sites to enhance the expression of ER molecular chaperone genes.
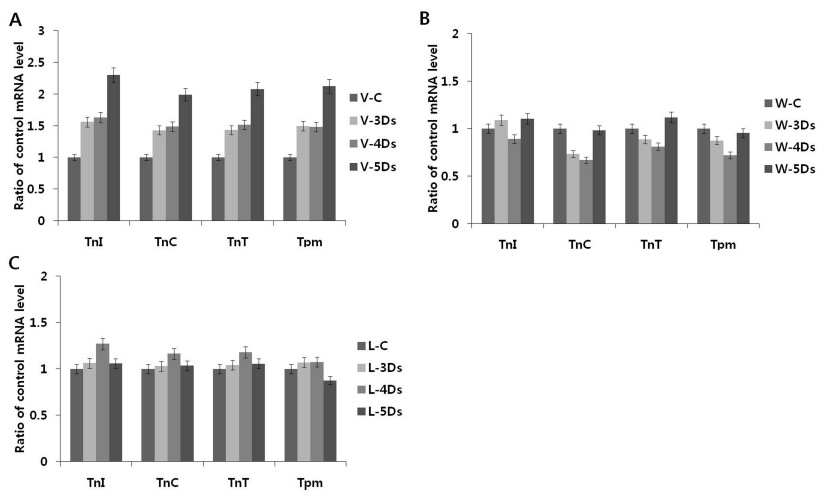

Figure 2. Results of gene expression of $\mathrm{TnC}, \mathrm{TnI}, \mathrm{Tn} T$, and Tpm from DVM, DWM, and DLM after starvation for three to five days. (A) Dorsal ventral flight muscle (DVM). Troponin I (TnI), Troponin C (TnC) Troponin T (TnT), and Tropomyosin (Tpm), V-C, DVM-control; V-3Ds, DVM-three days starvation; V-4Ds, DVM-four days starvation; V-5Ds, DVM-five days starvation. (B) Dorsal wing flight muscle (DWM). Troponin I (TnI), Troponin $C(\operatorname{TnC})$, Troponin $T$ (TnT), and Tropomyosin (Tpm). V-C, DWM-control; W-3Ds, DWMthree days starvation; $W-4 D s, D W M-$ four days starvation; $W-5 D$ s, DWM-five days starvation. (C) Dorsal longitudinal flight muscle (DLM). Troponin I (TnI), Troponin $C(\operatorname{TnC})$ Troponin $T(\operatorname{TnT})$, and Tropomyosin (Tpm). L-C, DLM-control; L-3Ds, DLM-three days starvation; $L-4 D$ s, $D L M$-four days starvation; $L-5 D s, D L M$-five days starvation. Data in graph are results of densitometry using four different samples per group.

Starvation induces activation of UPR. It is interesting to know how different skeletal muscles (DLM, DVM, and DWM) respond to ER stress in relation to starvation. After starvation for five days, skeletal muscles were tested for gene expression of ER chaperones (BiP, calreticulin, PDI) and an ER stress sensor of ATF6. As shown in Figures 3A-3C, significant gene expressions (at approximately two-fold higher) of ER chaperone and ATF6 were only observed in the DVM at three to five days after starvation compared to those in the control. This result is similar to results of gene expression of troponin complex and Tpm induced by starvation as described above in Figure 2. It is known that gene expression is up-regulated in the skeletal muscle under starvation conditions through concomitant expression of ARF4 and decorin which promotes proliferation of myoblast through an ER stress-related pathway [28]. Up-regulation of ATF4 mRNA is controlled by phosphorylation of eIF $2 \alpha$ induced by simulation of ER stress [29]. Short-term starvation stress (but not severe) has beneficial effects on various positive contributions of 
longevity, fungal resistance and climbing activity [30]. Therefore, we also examined gene expression of decorin, one of proteoglycan type myokines produced and released by skeletal muscle cells (Figure 3D). Starvation for five days (12 percent of cricket's life span of 42 days) induced ER stress, but not a strong stimulus to induce apoptosis. This suggests that relatively short-term starvation does not interfere with normal muscle physiology, including normal production of myokines such as decorin. However, if strong starvation persists, ER stress responder will induce abnormal muscle physiology through apoptosis.
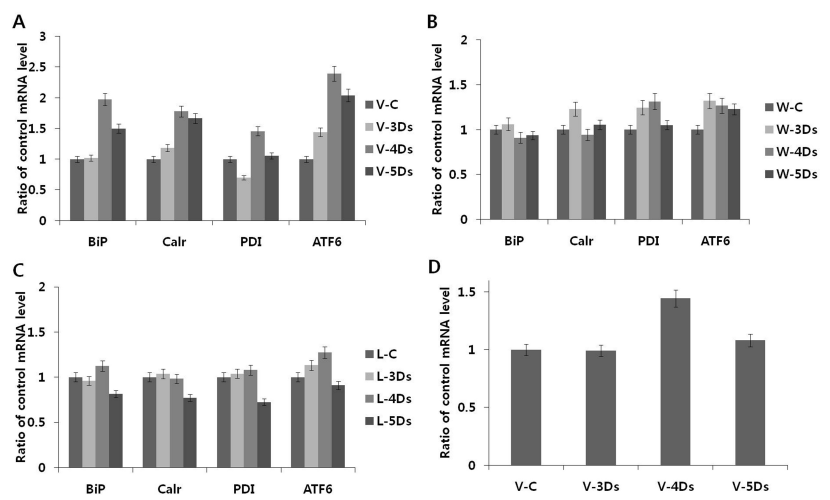

Figure 3. Results of gene expression of ER chaperones and decorin. (A) Dorsal ventral flight muscle (DVM). V-C, DVM-control; V-3Ds, DVM-three days starvation; V-4Ds, DVM-four days starvation; V-5Ds, DVM-five days starvation. Binding immunoglobulin protein (BiP), (Calr), disulfide isomerase (PDI), and ATF6. (B) Dorsal wing flight muscle (DWM). V-C, DVM-control; V-3Ds, DVM-three days starvation; V-4Ds, DVM-four days starvation; $V-5 D s, D V M$-five days starvation. Binding immunoglobulin protein (BiP), (Calr), disulfide isomerase (PDI), and ATF6. (C) Dorsal longitudinal flight muscle (DLM). L-C, DLM-control; L-3Ds, DLM-three days starvation; L-4Ds, DLM-four days starvation; L-5Ds, DLM-five days starvation. Binding immunoglobulin protein (BiP), (Calr), disulfide isomerase (PDI), and ATF6. (D) Results of decorin gene expression in DVM after starvation for three to five days. V-C, DVM-control; V-3Ds, DVM-three days starvation; V-4Ds, DVM-four days starvation; $V$-5Ds, DVM-five days starvation. Data in the graph are densitometry results for four different samples per group.

In summary, three kinds of muscles (DLM, DVM, and DWM) in a cricket of $G$. bimaculatus were commonly characterized by a typical skeletal muscle. Starvation only induced upregulation of gene expressions of ER chaperones (BiP, calreticulin, and PDI), ER transmembrane sensor of ATF6, troponin complex (TnC, TnI, and TnT), and tropomyosin (Tpm) in DVM, but not in DLM or DWM. DVM is affected by ER stress due to starvation. It produces decorin that is essential for skeletal muscle physiology. The precise mechanism of this result is unclear. Further experiments are needed. When studying gene expression affected by starvation, comparing three different skeletal muscles of the same physiological state within one individual is very important.

\section{Acknowledgement}

This study was supported by a grant from the Agenda program (PJ010864), Rural Development Administration, Republic of Korea.

\section{References}

1. Ahn MY, Hwang JS, Kim MJ, Park KK. Antilipidemic effects and gene expression profiling of the glycosaminoglycans from cricket in rats on a high fat diet. Arch Pharm Res 2016; 39: 26-936.

2. Nakamura T, Mito T, Bando T, Ohuchi H, Noji S. Dissecting insect leg regeneration through RNA interference. Cell Mol Life Sci 2008; 65: 64-72.

3. Febbraio MA, Pedersen BK. Contraction-induced myokine production and release: is skeletal muscle an endocrine organ? Exerc Sport Sci R 2005; 33: 114-119.

4. Pedersen BK, Febbraio MA. Muscles, exercise and obesity: skeletal muscle as a secretory organ. Nat Rev Endocrinol 2012; 8: 457-465.

5. Barone V, Randazzo D, Del Re V, Sorrentino V, Rossi DJ. Organization of junctional sarcoplasmic reticulum proteins in skeletal muscle fibers. Muscle Res. Cell Motil 2015; 36: 501-515.

6. Manstein DJ, Mulvihill DP. Tropomyosin-mediated regulation of cytoplasmic myosins. Traffic 2016; 17: 872-877.

7. Kalyva A, Parthenakis FI, Marketou ME, Kontaraki JE, Vardas PE. Biochemical characterisation of troponin $\mathrm{C}$ mutations causing hypertrophic and dilated cardiomyopathies. J. Muscle Res. Cell Motil 2014; 35: 161-178.

8. Sheng JJ, Jin JP. TNNI1, TNNI2 and TNNI3: Evolution, regulation, and protein structure-function relationships. Gene 2016; 576: 385-394.

9. Fahim MA, Hayen AD, Horvath AR, Dimeski G, Coburn A, Tan KS, Johnson DW, Craig JC, Campbell SB, Hawley CM. Biological variation of high sensitivity cardiac troponin-T in stable dialysis patients: implications for clinical practice. Clin Chem Lab Med 2015; 53: 715-722.

10. Squire JM, Paul DM, Morris EP. Myosin and actin filaments in muscle: Structures and interactions. Subcell Biochem 2017; 82: 319-371.

11. Oliver RH, Albury AN, Mousseau TA. Programmed cell death in flight muscle histolysis of the house cricket. J Insect Physiol 2007; 53: 30-39.

12. Baiceanu A, Mesdom P, Lagouge M, Foufelle F. Endoplasmic reticulum proteostasis in hepatic steatosis. Nat Rev Endocrinol 2016; 124: 710-722.

13. McCaffrey K, Braakman I. Protein quality control at the endoplasmic reticulum. Essays Biochm 2016; 60: 227-235.

14. Grootjans J, Kaser A, Kaufman RJ, Blumberg RS. The unfolded protein response in immunity and inflammation. Nat Rev Immunol 2016; 16: 469-484. 
15. Zhang L, Luo L, Jiang $X$. Starvation influences allatotropin gene expression and juvenile hormone titer in the female adult oriental armyworm, Mythimna separata. Arch Insect Biochem Physiol 2008; 68: 63-70.

16. Price DP, Schilkey FD, Ulanov A, Hansen IA. Small mosquitoes, large implications: crowding and starvation affects gene expression and nutrient accumulation in Aedes aegypti. Parasit Vectors 2015; 8: 252.

17. Bos N, Pulliainen U, Sundström L, Freitak D. Starvation resistance and tissue-specific gene expression of stressrelated genes in a naturally inbred ant population. $\mathrm{R}$ Soc Open Sci 2016; 3: 160062.

18. Petek M, Rotter A, Kogovšek P, Baebler S, Mithöfer A, Gruden K. Potato virus Y infection hinders potato defence response and renders plants more vulnerable to Colorado potato beetle attack. Mol Ecol 2014; 23: 5378-5391.

19. Tsintzas K, Jewell K, Kamran M, Laithwaite D, Boonsong T, Littlewood J, Macdonald I, Bennett A. Differential regulation of metabolic genes in skeletal muscle during starvation and refeeding in humans. J Physiol 2006; 575: 291-303.

20. Konuma $\mathrm{T}$, Tsukamoto $\mathrm{Y}$, Nagasawa $\mathrm{H}$, Nagata $\mathrm{S}$. Imbalanced hemolymph lipid levels affect feeding motivation in the two-spotted cricket, Gryllus bimaculatus. PLoS One 2016; 11: e0154841.

21. Werely WA. Automated hematoxylin and eosin staining for large volumes of tissue. Am J Med Technol 1976; 42: 285-287.

22. Rai N, Nongthomba U, Grounds MD. Skeletal muscle degeneration and regeneration in mice and flies. Curr Top Dev Biol 2014; 108: 247-281.

23. Lockshin RA, Colon AD, Dorsey AM. Control of muscle proteolysis in insects. Fed Proc 1980; 39: L4852.

24. Gubb D, Sanz-Parra A, Barcena L, Troxler L, Fullaondo A. Protease inhibitors and proteolytic signaling cascades in insects. Biochimie 2010; 92: 1749-1759.
25. Gomi T, Okuda T, Tanaka S. Protein synthesis and degradation in flight muscles of adult crickets (Gryllus bimaculatus). J Exp Biol 1995; 198: 1071-1077.

26. Cox JS, Walter P. A novel mechanism for regulating activity of a transcription factor that controls the unfolded protein response. Cell 1996; 87: 391-404.

27. Back SH, Kaufman RJ. Endoplasmic reticulum stress and type 2 diabetes. Annu Rev Biochem 2012; 81: 767-793.

28. Sun L, Lu K, Liu H, Wang H, Li X, Yang C, Li L, Wang J. The effects of endoplasmic reticulum stress response on duck decorin stimulate myotube hypertrophy in myoblasts. Mol Cell Biochem 2013; 377: 151-161.

29. Thomas DB, Ronald CW. Eukaryotic initiation factor 2 phosphorylation and translational control in metabolism. Adv Nutr 2012; 3: 307-321.

30. Chidawanyika F, Nyamukondiwa C, Strathie L, Fischer K. Starvation and age on heat tolerance of the parthenium beetle (Coleoptera: Chrysomelidae) following dynamic and staticprotocols. PLoS One 2017; 12: e0169371.

\section{*Corresponding to}

O-Yu Kwon

Departments of Medical Science and Anatomy and Cell Biology

College of Medicine

Korea

Seung-Whan Kim

Department of Emergency Medicine

College of Medicine

Korea 